\title{
A High-Performance Line-Start Permanent Magnet Synchronous Motor Amended From a Small Industrial Three-Phase Induction Motor
}

\author{
W. Fei ${ }^{1.2}$, P. C. K. Luk ${ }^{1}$, J. Ma ${ }^{2}$, J. X. Shen ${ }^{2}$, and G. Yang ${ }^{2}$ \\ ${ }^{1}$ Department of Engineering Systems and Management, Cranfield University, Shrivenham, Wiltshire SN6 8LA, U.K. \\ ${ }^{2}$ College of Electrical Engineering, Zhejiang University, Hangzhou, Zhejiang 310027, China
}

\begin{abstract}
Small industrial three-phase induction motors (IMs) normally suffer from poor operational efficiency and power factor. This paper presents a high-performance line-start permanent magnet synchronous motor (LSPMSM) which is developed by simple modifications of an off-the-shelf small industrial three-phase IM with minimized additional costs. Two-dimensional (2-D) dynamic finite element analysis (FEA) models are employed to assess the machine performances, which are validated by comprehensive experimental results. The experimental comparisons between the amended LSPMSM and the original IM have indicated that significant improvements in efficiency and power factor can be achieved by the proposed LSPMSM.
\end{abstract}

Index Terms-Line-start induction machine, permanent magnet synchronous motor, power factor improvement, dynamic finite element analysis.

\section{INTRODUCTION}

$\mathbf{N}$ OWADAYS, small three-phase induction motors (IMs) are widely used in many industrial applications, such as pumps and fans. Although small IMs are very cost competitive, they normally suffer from relatively low operational efficiency and power factor. As public concern for the environment and global warming is ever increasing, the prospect of improving the energy efficiency of running the vast number of IMs currently installed in industry is of particular interest. With the introduction of legislation, the demand for higher efficiency motors is set to increase. Although small permanent magnet synchronous machines can achieve high operational efficiency and power factor, they lack the starting capability of the IM. The line-start permanent magnet synchronous motor (LSPMSM) has been developed to achieve both high operational efficiency and starting capability. The LSPMSM uses a rotor cage to develop the starting torque that pulls the motor into synchronism, and permanent magnets for the generation of synchronous torque at steady state. Since it operates essentially as a synchronous motor, the induced current and hence copper losses in the rotor cage are negligible. Besides, the motor operates at a high power factor as a conventional permanent magnet synchronous motor. This further reduces the stator current and the associated losses.

Earlier studies on LSPMSMs appear to be mainly in the higher power applications, in which large scale IMs have been used as a baseline for the machine design [1]. Although high efficiency and good power factor can be relatively easy to achieve with the optimization of machine parameters, large LSPMSMs experience significant disadvantages as the manufacture and assembly of large permanent magnets can be very cumbersome [2]. On the other hand, since small IMs suffer lower efficiency and power factor as a result of higher penalty factor of field excitation, there has been much interest in the application of permanent magnets into the rotor core of the IM to alleviate the excitation penalty and hence improve the efficiency [3]-[5]. Contrary to large LSPMSMs, it is much easier to assembly small permanent magnets into the rotor core of small IMs. Indeed, it is envisaged that optimized LSPMSMs can be developed by artful modifications of existing small IMs, so that significant resources in redesigning and restocking of new motors of higher operational efficiency can be saved.

In this paper, the performance of a LSPMSM, developed by modifying an off-the-shelf industrial three-phase IM Y $90 \mathrm{~S}-4$, is investigated. The motor is designed to have adequate starting capability when connected to the mains supply with a large load inertia, and high efficiency at steady-state synchronous speed. Two-dimensional (2-D) time-stepping finite element analysis (FEA) models are used to analyze the steady state and dynamic performance of the prototype motor. The FEA-predicted performance of the machine is validated by experimental results. Experimental comparisons show that the LSPMSM prototype has superior efficiency and power factor to the original IM Y 90 S- 4 .

\section{Prototype Configuration and Design}

The prototype is modified from the existing IM Y90S-4, with the original configuration being kept as much as possible to minimize extra costs. The prototype's stator uses the same lamination core as Y90S-4, the material of which is DR510-50. Also, the winding pattern is kept unchanged, but the copper wire size and coil turn number are adjusted to ensure the machine can achieve near unity power factor operation at full load. Generally, a good rotor design is crucial in meeting the critical requirements for a high-performance LSPMSM. In this study, a four-pole rotor is proposed. It uses inexpensive DR600-50 lamination and high-strength sintered rare earth permanent magnets $\mathrm{NdFe} 35 \mathrm{SH}$, which can deliver high-energy product even at high temperatures. Fig. 1 shows the cross section of the rotor design, and the prototype rotor. The outer diameter of proposed rotor is made slightly smaller than that of the IM Y90S-4 to ease assembly. However, the key design focus rests on the squirrel cage and the permanent magnets. To ensure good starting capability, the dimension and number of rotor slot are designed 


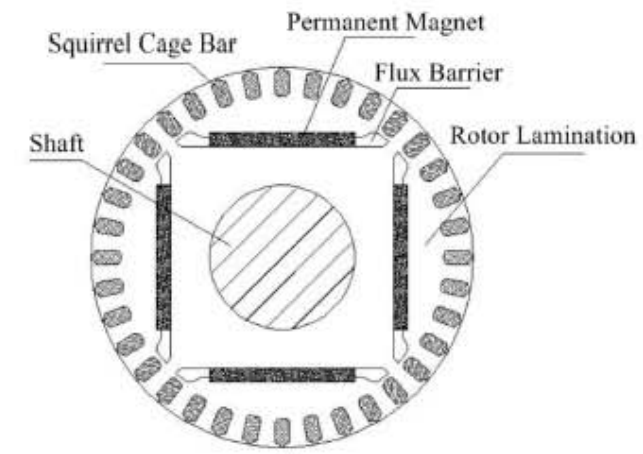

(a)

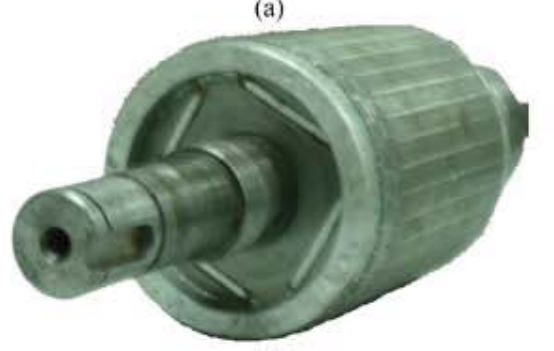

(b)

Fig. 1. Prototype rotor. (a) Configuration of the rotor. (b) Experimental rotor.

TABLE I

DESIGN DATA OF PROTOTYPE MOTOR

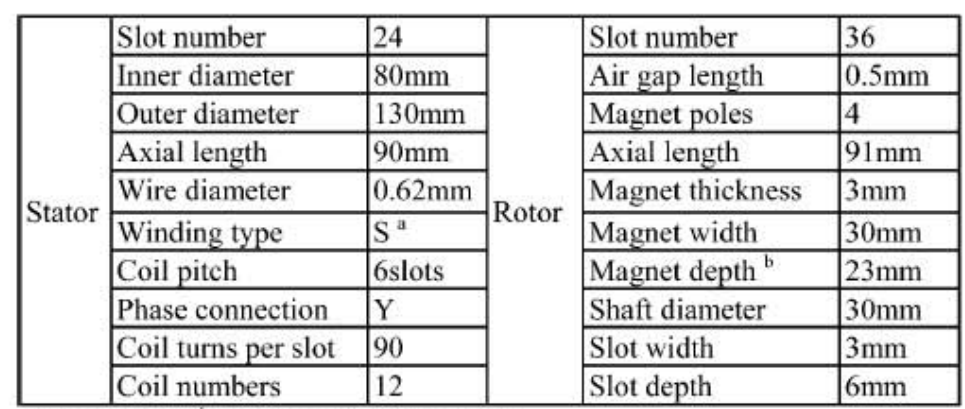

${ }^{a}$ Single layer, ${ }^{b}$ Magnet embedding depth

such that the back electromotive force (EMF) profile is close to sinusoidal, and cogging torque is to be sufficiently suppressed without resorting to rotor or stator skewing techniques. A rotor configuration of 36 pyriform slots with flat bottom, as shown in Fig. 1(a), is chosen to meet the requirements. The design of the permanent magnets depends on the required magnetic field profile and means of assembly. A design compromise is necessary to ensure ease of assembly and optimized use of magnet materials. It is determined that the interior-radial configuration with a cavity as the flux barrier at each end of the permanent magnets, as shown in Fig. 1(a), provides the optimum solution. The prototype rotor is shown in Fig. 1(b). The detailed design data for the proposed motor is presented in Table I.

\section{FEA Modeling AND EXPERIMENTAL VALIDATIONS}

Although analytical methods are employed widely for performance prediction of LSPMSMs, they do not normally yield results with a high level of accuracy. In this study, comprehensive 2-D time-stepped FEA models, developed by the commercial software ANSOFT MAXWELL-2D, are employed to accurately account for the effects of end windings, eddy current in the rotor bars and permanent magnets, and iron losses in the laminations. Fig. 2 shows the field distributions of the prototype with unloaded and full load conditions in the FEA models. The

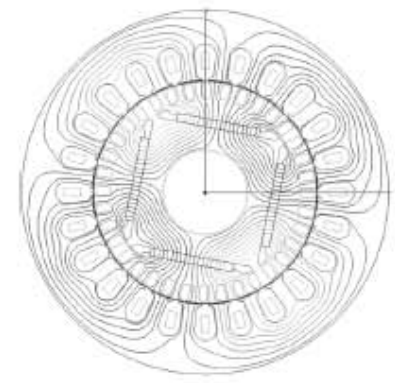

(a)

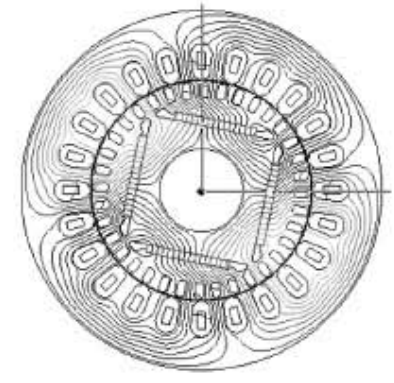

(b)
Fig. 2. Predicted field distribution of prototype. (a) Unloaded. (b) Full load.

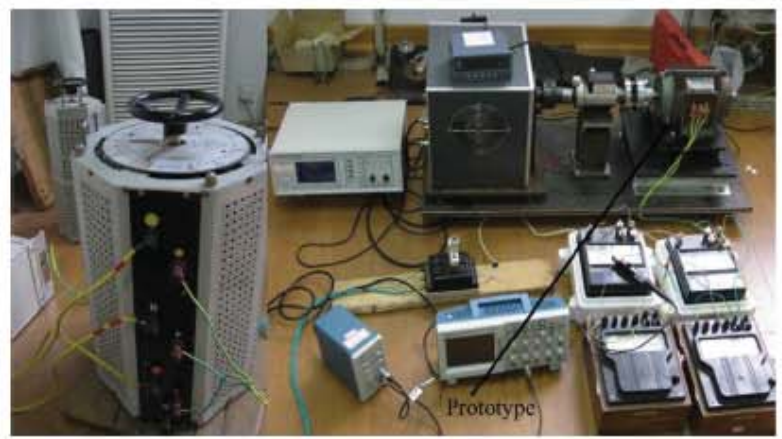

Fig. 3. Prototype and experimental setup.

models are also used to develop an improved analytical means for efficient performance predictions of the prototype motor. Furthermore, the prototype machine has been fabricated and tested to validate the predications from the FEA models. Fig. 3 shows the prototype motor and the experimental setup.

\section{A. Back EMF Induced by Permanent Magnets}

The back EMF induced by the permanent magnets under unloaded condition is a key parameter of the LSPMSM in meeting the simultaneous requirements of high efficiency and power factor during steady-state operations, and good starting capability during direct line-start conditions. The back EMF of the prototype can be determined simply by measuring its phase terminal voltage when the rotor is externally driven at the synchronous speed of $1500 \mathrm{rpm}$ without excitation in the winding coils. The mechanical transients can also be derived from the phase terminal voltage. Fig. 4 shows the FEA and experimental profiles of the phase back EMF, where excellent agreements are achieved. It can be seen that the slot openings on both the stator and rotor of the prototype, together with local saturations in the rotor due to magnetic leakage, inevitably give rise to nonlinearity and higher order harmonics, which result in a distorted sinusoidal back EMF.

\section{B. Steady-State Synchronous Performance}

Steady-state performance assessments normally consist of the armature phase current, efficiency and power factor under different load conditions, which can be calculated by justifiably neglecting the starting process and directly setting the rotor initial speed as synchronous speed of $1500 \mathrm{rpm}$ in the dynamic FEA models to significantly save computation time. Fig. 5 shows the simulated and measured armature phase current waveforms, which are in good agreements allowing for both measurement and simulation errors. Similarly, satisfactory 


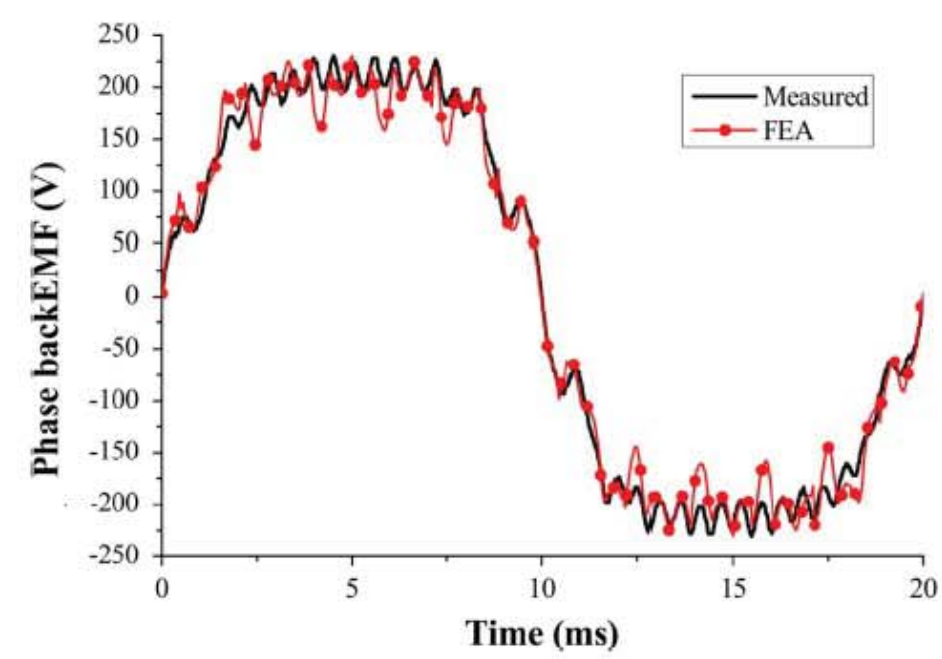

Fig. 4. Phase back EMF waveforms.

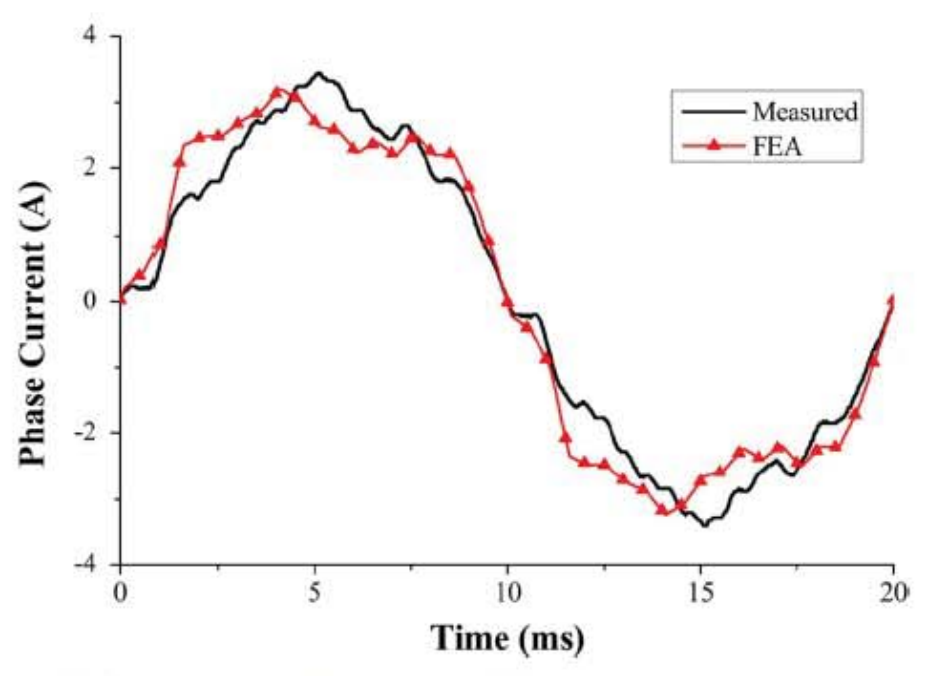

Fig. 5. Phase current waveforms under full load.

agreements are also achieved for different load conditions as shown in Fig. 6. Furthermore, the simulated and measured steady-state synchronous operation efficiencies under a wide load range also in close agreements, as illustrated in Fig. 7. It should be noted that due to the imperfections of the laminations, and the estimated loss coefficients provided by the manufacturer, it is always very difficult to evaluate iron losses with a high degree of confidence. This partly accounts for the discrepancy between the results. From Fig. 6, it is noted that the armature phase current tends to decrease and then increase when the prototype is loaded up, which is caused by insufficient flux linkage induced by permanent magnets in the winding. This will result in lower power factor and high copper losses under light load condition. This can be improved by increasing the winding flux linkage using higher number of turns of the machine winding.

\section{Dynamic Starting Performance}

The dynamic starting behavior of the LSPMSM is of great interest since the motor is known to have poor starting performance. Here, 2-D dynamic FEA models are employed to analyze the dynamic starting performance of the prototype. The stator is supplied with balanced three-phase voltages at rated frequency of $50 \mathrm{~Hz}$ and rated voltage of $220 \mathrm{~V}$. The inertia of the testing system is estimated to be $0.0026 \mathrm{~kg} \cdot \mathrm{m}^{2}$. Fig. 8 shows

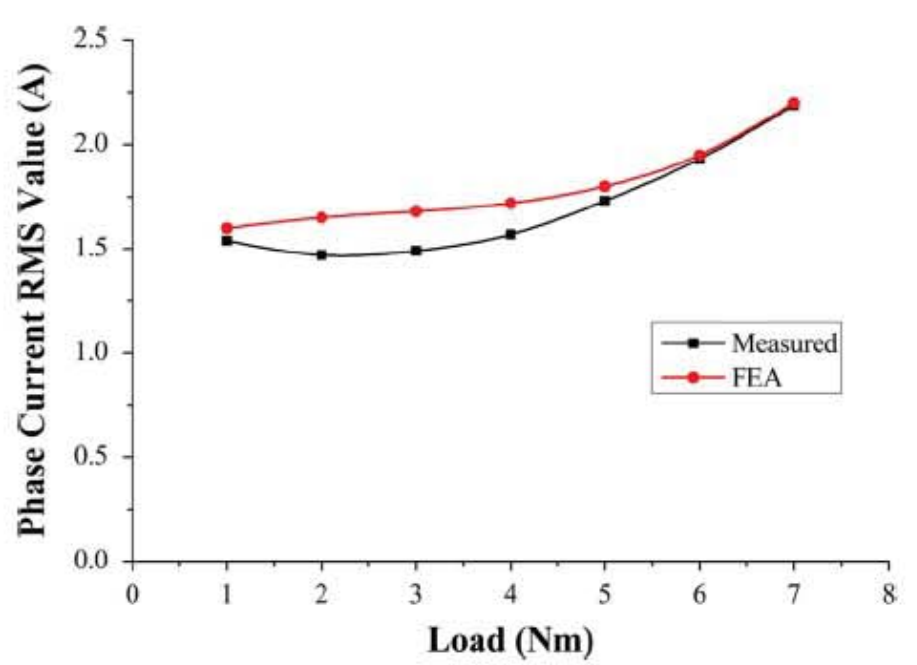

Fig. 6. Phase current RMS values at different loads.

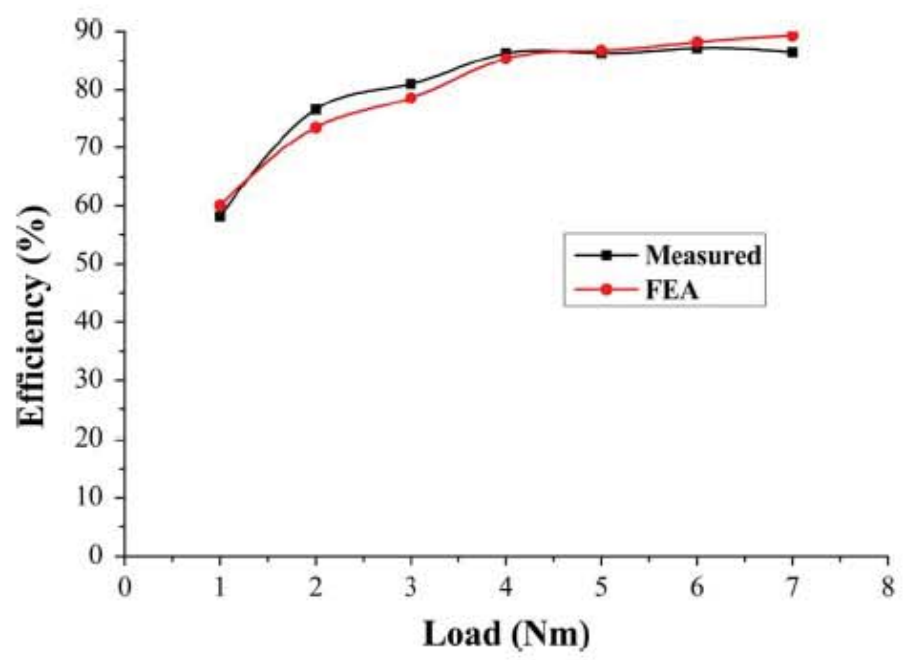

Fig. 7. Prototype efficiency at different loads.

the predicted dynamic responses when the motor is started direct-on-line over a range of inertia loads. Fig. 9 shows the experimental current waveforms of the prototype during the unloaded starting period. Both the simulated speed-time response profile and experimental current waveform show the motor is pulled into synchronism at around $0.21 \mathrm{~s}$ after the start on unloaded condition. It is also evident from Fig. 8 that the prototype can synchronize under an inertia load of up $200 \%$, but cannot do so when the inertia load is $300 \%$. From the result, it shows the prototype exhibits reasonable starting ability up to a $200 \%$ inertia overload. Beyond $200 \%$, it is likely that the motor will struggle to pull into synchronism, and will operate with increased copper losses in the rotor. It is worth mentioning that the starting capability of the prototype can be improved by increasing the depth of the rotor slots.

\section{Comparison Between Proposed LSPMSM AND ORIGINAL IM Y90S-4}

The steady-state performances of the original IM Y90S-4 and the prototype are compared. Fig. 10 shows the armature phase current at different loads between the two machines. It can be seen the armature current of the prototype is between $10-25 \%$ lower than that of the IM Y90S-4 for a given output. The comparison of the efficiency and power factor between the two motors under different load are shown in Table II. It can be seen 


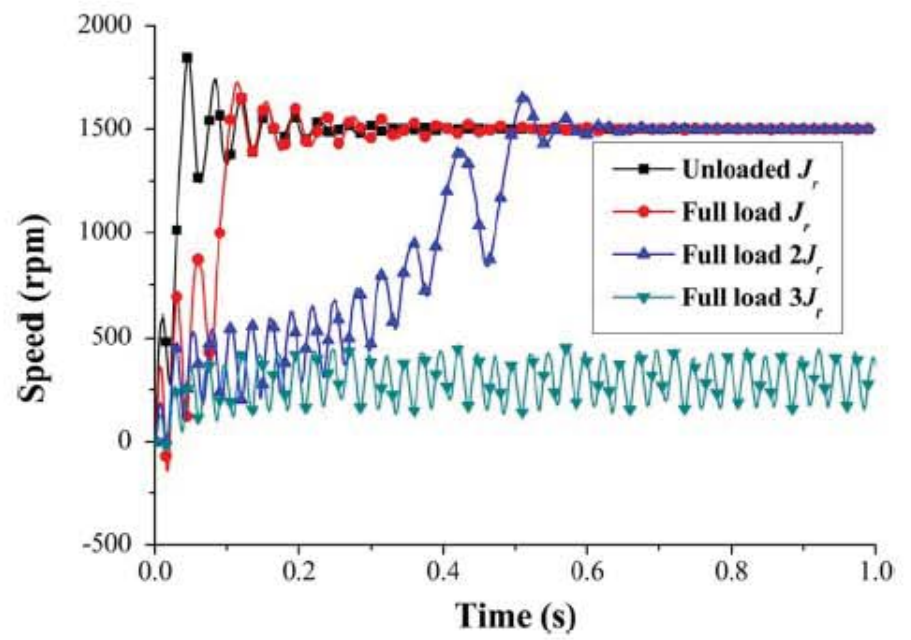

Fig. 8. Simulated speed-time responses.

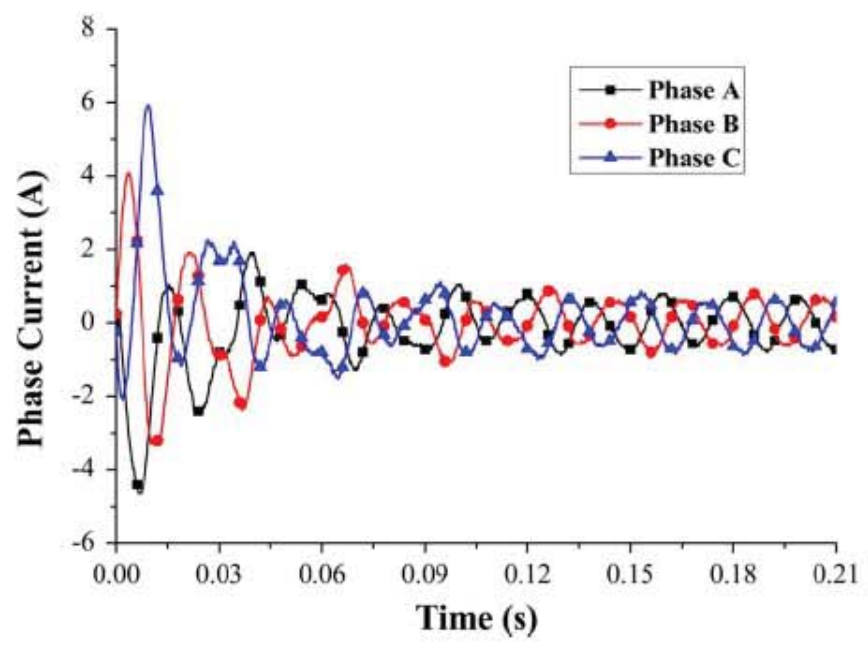

Fig. 9. Unloaded starting current waveforms from experiment.

that the prototype motor has higher efficiency and power factor over a wide range of load. Specifically, when the load is more than half the rated value, the efficiency of the prototype is about $7 \%$ higher than that of Y90S-4, and the power factor is about 0.14 higher. It is thus evident that the performance improvement of the prototype is quite remarkable given the relatively modest modification effort spent. On the other hand, the extra costs for the proposed LSPMSM are estimated to be around $20 \%$ of the cost of IM Y90S-4, which can be readily paid back by an equivalent of 550 hours of full load operation due to the savings in electricity bill.

\section{CONCLUSION}

A high-performance LSPMSM modified from a small industrial IM Y90S- 4 has been successfully developed and tested with minimum extra costs and development time. 2-D time-stepping FEA models have been employed to predict the steady-state and transient performances of the prototype motor. Experimental results are compared with the simulated results from the models.

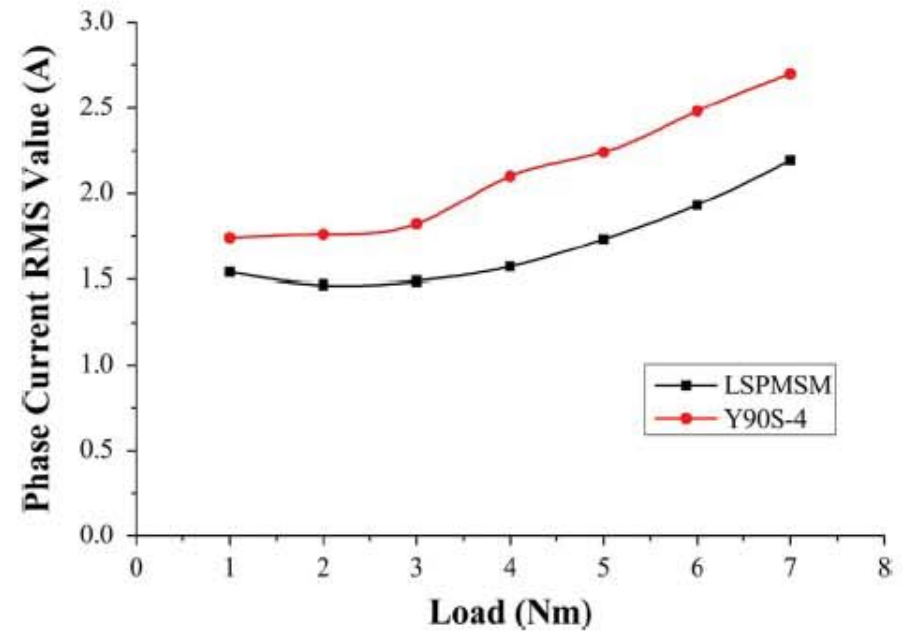

Fig. 10. Phase current RMS value comparison between proposed LSPMSM and IM Y90S-4 at different loads.

TABLE II

EFFICIENCY AND POWER FACTOR COMPARISON BETWEEN PROPOSED LSPMSM AND IM Y90S-4

\begin{tabular}{|c|c|c|c|c|}
\hline \multirow{2}{*}{ Load (N.m) } & \multicolumn{2}{|c|}{ Proposed LSPMSM } & \multicolumn{2}{c|}{ IM Y90S-4 } \\
\cline { 2 - 5 } & Efficiency (\%) & Power factor & Efficiency (\%) & Power factor \\
\hline 1 & 58.2 & 0.264 & 55.7 & 0.244 \\
\hline 2 & 76.6 & 0.422 & 70.5 & 0.379 \\
\hline 3 & 81.0 & 0.589 & 77.0 & 0.498 \\
\hline 4 & 86.3 & 0.707 & 78.4 & 0.564 \\
\hline 5 & 86.3 & 0.793 & 79.8 & 0.643 \\
\hline 6 & 87.2 & 0.851 & 79.1 & 0.696 \\
\hline 7 & 86.5 & 0.882 & 79.6 & 0.736 \\
\hline
\end{tabular}

The good agreements between the results confirm the validity of the FEA models. The comparisons between the proposed prototype and original Y90S-4 based on experimental tests have demonstrated that much improved efficiency and power factor are achieved with minimum modification efforts. Significant energy can be saved without substantial additional investments if the Y90S-4 IMs are modified in large scale by the proposed LSPMSM.

\section{REFERENCES}

[1] M. A. Rahman and A. M. Osheba, "Performance of large line-start permanent magnet synchronous motors," IEEE Trans. Energy Convers., vol. 5, no. 1, pp. 211-217, Mar. 1990.

[2] Q. F. Lu and Y. Y. Ye, "Design and analysis of large capacity linestart permanent-magnet motor," IEEE Trans. Magn., vol. 44, no. 11, pp. 4417-4430, Nov. 2008.

[3] G. Yang, J. Ma, J. X. Shen, and Y. Wang, "Optimal design and experimental verification of line-start permanent magnet synchronous motor," in Proc. Int. Conf. Electrical Machine and Syst., 2008, pp. 3232-3236.

[4] A. M. Knight and C. I. McClay, "The design of high efficiency line-start motors," IEEE Trans. Ind. Applicat., vol. 36, no. 6, pp. 1555-1562, Nov./Dec. 2000.

[5] K. Kurihara and M. A. Rahman, "High-efficiency line-start interior permanent-magnet synchronous motors," IEEE Trans. Ind. Applicat., vol. 40, no. 3, pp. 789-796, May/Jun. 2004. 